\title{
Gerontology
}

\section{New Trend in Old-Age Mortality: Gompertzialization of Mortality Trajectory}

\author{
Leonid A. Gavrilov Natalia S. Gavrilova \\ Academic Research Centers, NORC at the University of Chicago, Chicago, IL, USA
}

\section{Keywords}

Age misreporting · Biodemography - Gompertz law · Longevity $\cdot$ Mortality deceleration $\cdot$ Old age $\cdot$ Kannisto model $\cdot$ Late-life mortality

\begin{abstract}
There is great interest among gerontologists, demographers, and actuaries in the question concerning the limits to human longevity. Attempts at getting answers to this important question have stimulated many studies on late-life mortality trajectories, often with opposing conclusions. One group of researchers believes that mortality stops growing with age at extreme old ages, and that hence there is no fixed limit to the human life span. Other studies found that mortality continues to grow with age up to extreme old ages. Our study suggests a possible solution to this controversy. We found that mortality deceleration is best observed when older, less accurate life span data are analyzed, while in the case of more recent and reliable data there is a persistent mortality growth with age. We compared the performance (goodness of fit) of two competing mortality models - the Gompertz model and the Kannisto ("mortality deceleration") model - at ages of 80-105 years using data for 1880-1899 single-year birth cohorts of US men and women. The mortality modeling approach suggests a transition from mortality
\end{abstract}

deceleration to the Gompertzian mortality pattern over time for both men and women. These results are consistent with the hypothesis about disappearing mortality deceleration over time due to improvement in the accuracy of age reporting. In the case of more recent data, mortality continues to grow with age even at very old ages. This observation may lead to more conservative estimates of future human longevity records.

(c) 2019 S. Karger AG, Basel

\section{Introduction}

There is great interest among gerontologists, demographers, and actuaries in the question concerning the limits to human longevity, and whether any fixed limits exist at all. Attempts at getting answers to these important questions have stimulated many studies on late-life mortality trajectories, often with opposing conclusions. One group of researchers claims that mortality stops growing with age at extreme old ages, and that hence there is no fixed limit to the human life span [1]. Other studies found that mortality continues to grow with age even in late life [2]. Recent debates in Gerontology about limits to the human life span have highlighted the need to know better the shape of mortality trajectories at extreme old ages $[3,4]$.

\section{KARGER}

(c) 2019 S. Karger AG, Basel

E-Mail karger@karger.com

www.karger.com/ger
Leonid A. Gavrilov

NORC at the University of Chicago

1155 E 60th Street

Chicago, IL 60637 (USA)

E-Mail gavrilov@longevity-science.org 
It has long been believed that the exponential growth of the force of mortality with age (the Gompertz law) is followed by a period of deceleration in which mortality growths more slowly than predicted by the Gompertz law [5-8]. Thatcher [7] and Thatcher et al. [8] tested several models of mortality using data from 13 countries with a presumably good quality of mortality statistics and found downward deviation of mortality from the Gompertz law after the age of 80 years. Horiuchi and Wilmoth [6] analyzed age trajectories of life table aging rates in Sweden and Japan and found that the life table aging rate has a tendency to decline after 75-80 years of age, suggesting mortality deceleration. This mortality deceleration eventually produces "late-life mortality leveling-off" and "latelife mortality plateaus" at extreme old ages $[5,9,10]$. Actuaries (including Gompertz himself) first noted this phenomenon, and they proposed a logistic formula for mortality growth with age in order to account for mortality deceleration at advanced ages. The same phenomenon of "almost non-aging" survival dynamics at extreme old ages was observed in other biological species. In some species (medflies and house flies) the mortality plateau can occupy a sizable part of their life [11]. The existence of mortality plateaus is now well documented for a number of lower organisms [see review in 11]. However, later studies have reported no mortality deceleration at older ages for primates $[12,13]$, rodents, and humans $[2,14]$. In another study, Gompertz-like mortality was found for Australia, Canada, and the USA and mortality deceleration for a number of European countries [15].

Estimation of the force of mortality at very old ages causes difficulties, because of the very small number of survivors to these ages and because of age misreporting by older persons. Age misreporting may be a problem affecting estimates of mortality at older ages [16-19]. Even a small percentage of inaccurate data can greatly distort mortality trajectories at advanced ages $[20,21]$. In most cases, age misreporting at older ages leads to mortality underestimation [22], and even rare errors in age reporting can accumulate at extreme old ages, leading to spurious mortality deceleration [21]. Taking into account that the accuracy of age reporting is positively correlated with education [23], we can hypothesize that improvement of education over time would lead to more accurate age reporting and hence less prevalent mortality deceleration. Also, it is known that birth registration in the USA was introduced gradually, with only 6 states having official birth registration between 1841 and 1890 [24]. Introduction of birth registration improves the quality of age reporting among subsequent birth cohorts. Preliminary data suggest that mortality at advanced ages in earlier US birth cohorts shows stronger deceleration than mortality in later birth cohorts [14].

Here we analyze the historical evolution of mortality trajectories at advanced ages using age-specific death rates for 20 cohorts born in 1880-1899 in the USA. We study two competing models describing mortality trajectories (the Gompertz model and the Kannisto model) with the Akaike goodness-of-fit criterion. We hypothesize that mortality deceleration should fade away in more recent birth cohorts due to improvement in age reporting over time.

\section{Data and Methods}

The Human Mortality Database (HMD) provides mortality and population data [25]. Age-specific death rates are used as empirical estimates of the force of mortality [26]. Data sets of agespecific cohort death rates (central mortality rates) of men and women are available from the HMD for ages up to 110 years or older. We selected data available in 1-year age and time increments, denoted as $M_{x}$, where $x$ indicates a single year of age. The yearly central death rate for cohort $c$ at age $x$ is defined as the number of deaths in cohort $c$ between ages $x$ and $x+1$ divided by the number of person-years lived by cohort $c$ between ages $x$ and $x+$ 1 [27]. We studied 1880-1899 single-year birth cohorts from the USA and analyzed the data for men and women separately.

A study on data quality at older ages found that US data quality is conditionally acceptable [28]. The US data passed the tests of age heaping and Whipple's index for centenarians, but consistently showed moderate data quality problems [28]. Age-specific femaleto-male ratios confirmed that the US data were of sufficiently good quality up to the age of 106-107 years [14]. However, age misreporting was rapidly increasing after the age of 105 years [18, 29]. For this reason, mortality was fit in the age interval of $80-105$ years.

We fit mortality with two competing models used earlier in the study by Thatcher et al. [8]: the Gompertz model and the logistic model (a simplified two-parameter logistic model, also called the Kannisto model):

$$
\text { Gompertz: } \mu(x)=a e^{b x}
$$

and

$$
\text { Kannisto: } \mu(x)=\frac{a e^{b x}}{1+a e^{b x}},
$$

where $\mu(x)$ is the force of mortality, $x$ is age, and $a$ and $b$ are parameters.

Parameters $a$ and $b$ are called the intercept and the slope, respectively. Parameter $a$ determines the initial level of mortality, while parameter $b$ determines the rate of mortality increase with age.

According to the Gompertz law (suggested by the British actuary Benjamin Gompertz in 1825), the logarithm of the force of mortality (hazard rate) increases linearly with age. This is often used in order to graphically illustrate the validity of the Gompertz law - the data are plotted in the semi-log scale (known as the Gom- 
Fig. 1. Changes in Akaike information criterion (AIC) across birth cohorts for the Gompertz and the Kannisto model fitting US mortality. Lower AIC values correspond to a better model fit.

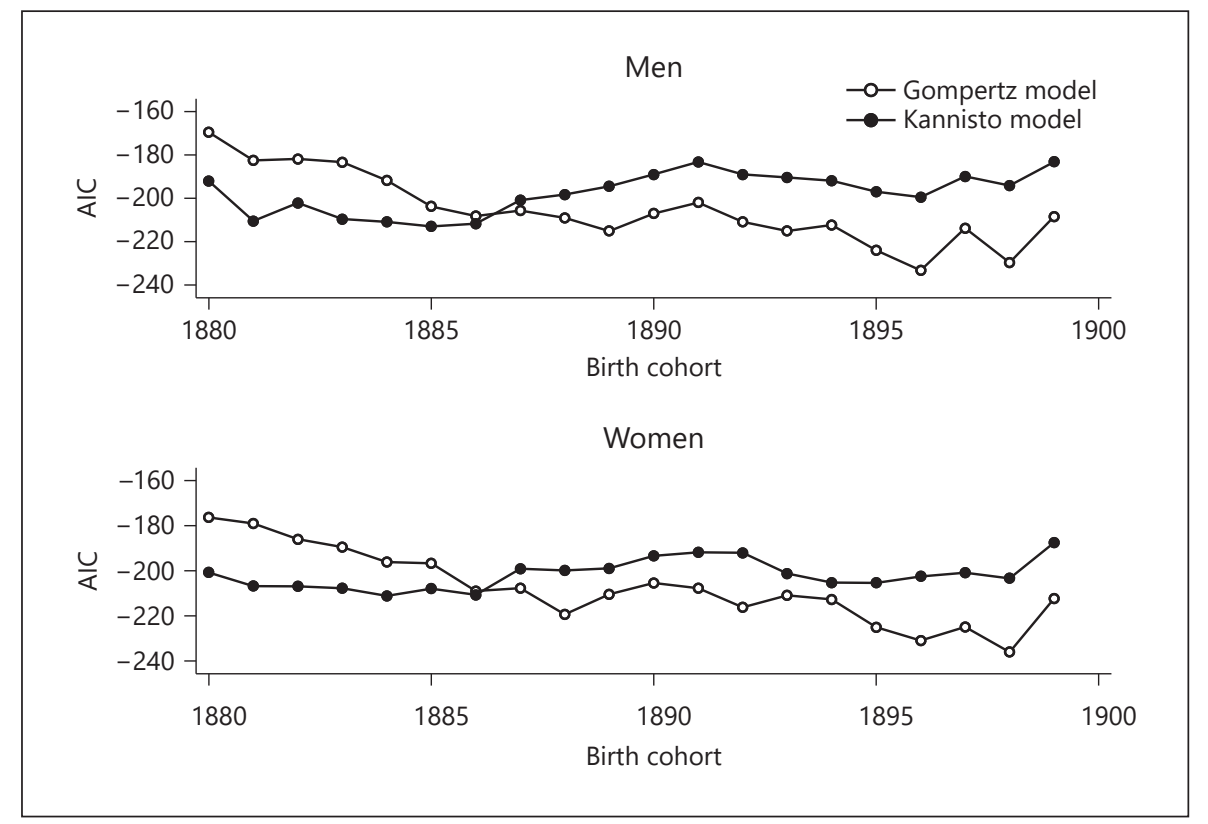

pertz plot) to check whether the logarithm of the hazard rate is indeed increasing with age in a linear fashion.

The Kannisto model was suggested by Väinö Kannisto in 1994 in order to take into account the observed mortality deceleration at advanced ages [30]. With the logistic Kannisto model, there is an asymptote of mortality at the oldest ages equal to 1 , and hence the Kannisto model fits mortality better in the case of mortality deceleration.

We tested these two models for their performance in fitting the empirical data. We ran a weighted nonlinear regression model in the age interval of 80-105 years (using the Stata command nlin). Age-specific exposure values were used as weights in the nonlinear regression analyses [31].

The Akaike information criterion (AIC) was used to evaluate goodness of fit for the Gompertz and the Kannisto model:

$$
A I C=2 k-2 \ln (L)
$$

where $\ln (L)$ is the maximized log-likelihood of the model and $k$ is the number of parameters estimated. AIC was computed using the Stata postestimation command estat. Both the nlin and estat commands are described in the Stata manual [32].

The best model has the minimal value of the AIC [33]. It is not the absolute size of the AIC value, it is the difference in values for the compared models $\left(\Delta_{i}\right)$ that is important in model selection. An AIC difference $>10$ suggests strong support for a better model by the data [33]:

$$
\Delta_{i}=A I C_{i}-A I C_{\text {min }}
$$

where $\Delta_{i}$ is the AIC difference for the $i$-th model, $A I C_{i}$ is the AIC value for the $i$-th model, and $A I C_{\text {min }}$ is the value of the criterion for the best model with the minimal AIC.

The analyses were conducted using Stata statistical software, release 14 .

New Trend in Old-Age Mortality

\section{Results}

Figure 1 presents the results of model fitting (AIC values) for the US 1880-1899 birth cohorts. It demonstrates a clear transition from lower AIC values (better fit) for the Kannisto model in the case of earlier birth cohorts to lower AIC values (better fit) for the Gompertz model in the case of later birth cohorts. This phenomenon means that mortality deceleration, which is observed for both men and women in earlier birth cohorts, disappears in more recent birth cohorts. This historical change in the pattern of mortality trajectories from mortality deceleration to the Gompertz law occurs for persons born around 18861887 (Fig. 1).

Table 1 presents the changes in AIC and AIC difference for the subsequent birth cohorts. Note that in most cases, the AIC difference was $>10$, suggesting strong support by the data for either the Kannisto model (in the case of the earlier cohorts) or the Gompertz model (in the case of the later cohorts). Thus, we may conclude that mortality in the past indeed demonstrated deceleration, whereas later it changed to the Gompertz pattern.

Figure 2 illustrates the historical changes in mortality trajectories, presenting age-specific mortality on a semilog scale for an earlier (1881) and a later (1898) birth cohort of women in the USA. The mortality pattern of the earlier birth cohort demonstrated an obvious deceleration of mortality growth with age, while the mortality pattern of the later birth cohort presented a persistent growth 


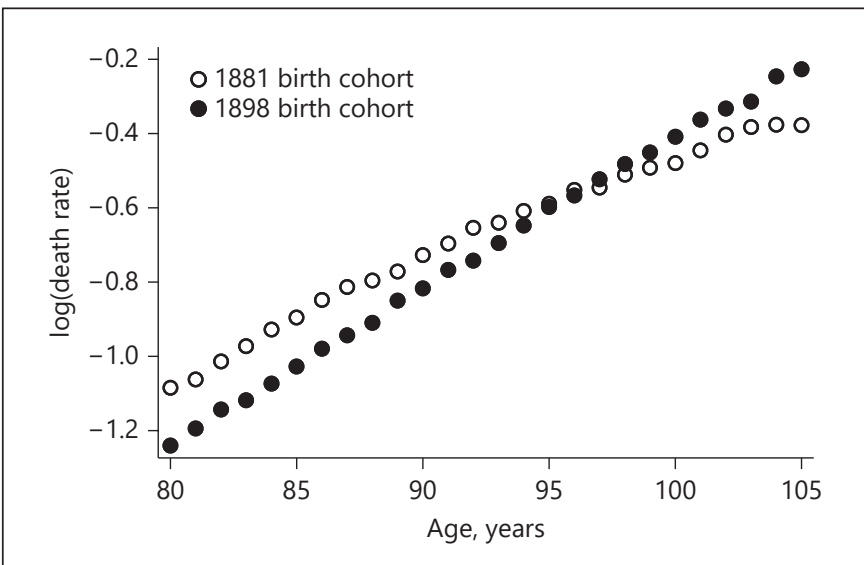

Fig. 2. Mortality (death rate) as a function of age for an earlier (1881) and a later (1898) birth cohort of US women.

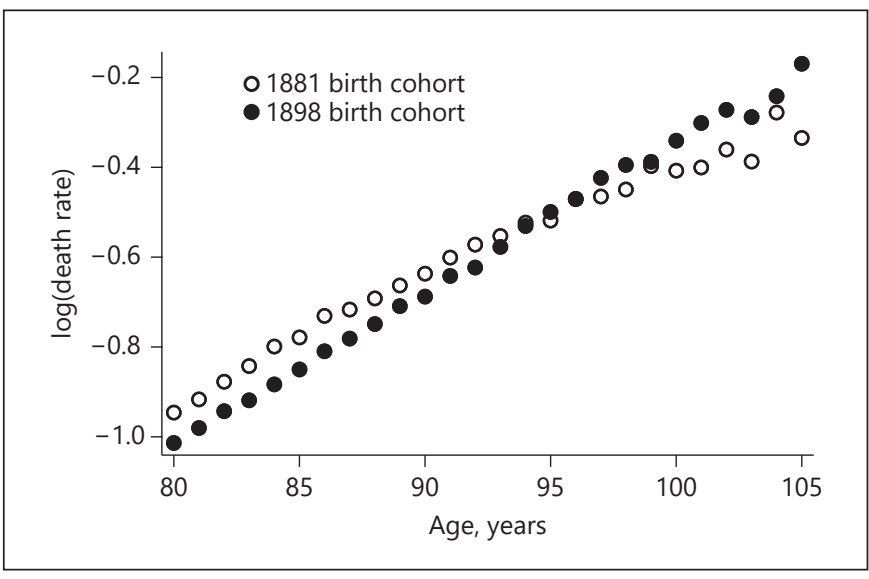

Fig. 3. Mortality (death rate) as a function of age for an earlier (1881) and a later (1898) birth cohorts of US men.

Table 1. Testing two competing models: Akaike information criterion (AIC) and AIC differences for the Gompertz and the Kannisto model

\begin{tabular}{|c|c|c|c|c|c|c|}
\hline \multirow{2}{*}{$\begin{array}{l}\text { Birth } \\
\text { cohort }\end{array}$} & \multicolumn{3}{|l|}{ Men } & \multicolumn{3}{|l|}{ Women } \\
\hline & $\begin{array}{l}\text { Gompertz } \\
\text { model (1) }\end{array}$ & $\begin{array}{l}\text { Kannisto } \\
\text { model (2) }\end{array}$ & $\begin{array}{l}\text { AIC difference, } \\
\Delta_{\mathrm{i}}(1)-(2)\end{array}$ & $\begin{array}{l}\text { Gompertz } \\
\text { model (1) }\end{array}$ & $\begin{array}{l}\text { Kannisto } \\
\text { model (2) }\end{array}$ & $\begin{array}{l}\text { AIC difference, } \\
\Delta_{\mathrm{i}}(1)-(2)\end{array}$ \\
\hline 1880 & -169.55 & -192.07 & 22.52 & -176.34 & -200.78 & 24.44 \\
\hline 1881 & -182.52 & -210.46 & 27.94 & -179.01 & -206.80 & 27.79 \\
\hline 1882 & -181.83 & -202.12 & 20.29 & -185.98 & -206.89 & 20.91 \\
\hline 1883 & -183.31 & -209.56 & 26.25 & -189.48 & -207.74 & 18.26 \\
\hline 1884 & -191.75 & -210.89 & 19.14 & -196.12 & -211.12 & 15.00 \\
\hline 1885 & -203.78 & -212.98 & 9.20 & -196.67 & -207.93 & 11.26 \\
\hline 1886 & -208.18 & -211.71 & 3.53 & -208.96 & -210.66 & 1.70 \\
\hline 1887 & -205.67 & -200.85 & -4.83 & -207.71 & -199.09 & -8.62 \\
\hline 1888 & -209.03 & -198.26 & -10.77 & -219.34 & -199.84 & -19.50 \\
\hline 1889 & -215.13 & -194.48 & -20.65 & -210.41 & -198.90 & -11.51 \\
\hline 1890 & -207.04 & -188.96 & -18.07 & -205.40 & -193.38 & -12.02 \\
\hline 1891 & -201.96 & -183.22 & -18.74 & -207.72 & -191.80 & -15.92 \\
\hline 1892 & -210.89 & -188.96 & -21.92 & -216.20 & -192.00 & -24.20 \\
\hline 1893 & -215.09 & -190.38 & -24.71 & -210.89 & -201.20 & -9.70 \\
\hline 1894 & -212.31 & -191.89 & -20.42 & -212.68 & -205.22 & -7.46 \\
\hline 1895 & -223.97 & -196.96 & -27.02 & -225.02 & -205.37 & -19.65 \\
\hline 1896 & -233.26 & -199.47 & -33.80 & -230.94 & -202.51 & -28.43 \\
\hline 1897 & -213.76 & -189.90 & -23.86 & -224.90 & -200.85 & -24.05 \\
\hline 1898 & -229.67 & -194.13 & -35.54 & -236.07 & -203.37 & -32.70 \\
\hline 1899 & -208.38 & -183.20 & -25.18 & -212.28 & -187.50 & -24.77 \\
\hline
\end{tabular}

Smaller AIC values for the Gompertz model and negative values of AIC difference correspond to better fit of the Gompertz model.

(straight line). Figure 3 demonstrates similar mortality patterns for an earlier and a later birth cohort of US men.

Note that above the age of 95 years, the mortality estimates for the earlier birth cohort were even lower than the mortality estimates for the later birth cohort. This paradoxical observation is most likely related to a better quality of age reporting among more recent birth cohorts. 


\section{Discussion}

This study of 1880-1899 single-year birth cohorts found that mortality deceleration is more prevalent in historically earlier birth cohorts, while more recent birth cohorts tend to demonstrate the Gompertzian pattern of mortality. These results support the hypothesis that mortality deceleration disappears over time apparently due to improvement in age reporting. These results agree with previous reports of Gompertzian mortality in the US cohorts born after 1889 [2, 14].

Our results may help to understand why earlier studies had found mortality deceleration and mortality leveling-off $[6-8,30]$, while more recent studies did not confirm these initial findings $[2,14]$. More frequent age misreporting in the past by older individuals is one of the most likely reasons for this phenomenon $[14,21,34]$. Studies conducted more than 20 years ago used data for earlier birth cohorts, when age reporting was not particularly accurate [28]. A later study [15] found that oldage mortality in Australia, Canada, and the USA is compatible with the Gompertzian model, confirming our findings.

Age misreporting in the USA could be particularly prevalent, because this country did not have an established uniform system of birth registration up to 1933 [24]. For example, Kestenbaum and Ferguson [35] were able to find only 52 birth certificates of long-lived persons out of 325, whereas the age of 256 persons was validated through the early US censuses. According to our experience, age misreporting indeed is able to produce false mortality deceleration [11]. We conducted a direct age validation procedure for records of persons born in 1900 and aged 106 years and over by linking data available from the US Social Security Administration Death Master File to early historical resources, including early censuses. We found that data cleaning (removing records with unconfirmed age) resulted in higher mortality estimates at advanced ages and more Gompertz-like mortality trajectories [11]. The proportion of records with unconfirmed age increases with age from $12 \%$ at the age of 100 years to $17 \%$ at age 105 years and 35\% at ages of 109 years and over [18]. The advantage of studying US mortality is the large population size of the USA with a significant number of survivors to advanced ages, which allows researchers to obtain more reliable estimates of the force of mortality (hazard rate).

In addition to age misreporting, some other causes of the transition from late-life mortality deceleration to Gompertzian mortality may be involved. It was recently observed that the mortality of centenarians in the USA had not decreased noticeably in the past decades, despite a significant decline in mortality among younger age groups [3]. In some countries (Japan, France, Switzerland, and Sweden), the historical decline in mortality among centenarians had stopped about $10-20$ years ago [36]. Historical stagnation of mortality at ages of 100 years and older may lead to steeper mortality curves for cohorts at extreme old ages, while a historical mortality decline after the age of 100 years may produce an apparently decelerating pattern of mortality with age. Feehan [37] studied cohort mortality after the age of 80 years in different countries. He found that in some countries the Gompertz model performed poorly, while in other countries this model performed reasonably well. He suggested that period effects may have altered the shape of cohort mortality, producing the strongest evidence for non-Gompertzian death rates for cohorts that experienced continuous historical improvements in mortality at advanced ages [37]. Our results do not completely exclude the possibility that the age at onset of mortality deceleration in the USA has moved beyond 105 years of age. Indeed, it was found that mortality in European countries does show deceleration, but the age at onset of mortality deceleration is shifting over time to older ages [15]. Thus, the same process could happen in the USA too.

It is possible that both the improvement of age reporting and stagnation of mortality among centenarians contribute to the observed historical transition from mortality deceleration to the Gompertzian pattern. Overall, it appears that the onset of mortality deceleration now occurs at much older ages than those reported earlier $[6-8,38]$.

Also, there are theoretical models $[39,40]$ and simulation studies [41] suggesting that mortality deceleration at advanced ages may be a consequence of population heterogeneity. The heterogeneity hypothesis predicts that the age at onset of mortality deceleration should increase over time as frailer individuals reach more advanced ages due to lower mortality at younger ages $[6,42]$. Studies of the US period mortality [42] and cohort mortality in other countries $[6,15]$ provide some empirical support for this prediction. A study of the US period mortality revealed an expansion of life span inequalities over time among survivors to older ages, suggesting that more heterogeneous populations are reaching older age now [43]. 


\section{Conclusions}

Our results demonstrate that there is no single universal answer to the question concerning the mortality pattern at extreme old ages, because this answer depends on the historical period of mortality analysis. In old historical data, late-life mortality deceleration is observed. In more recent data, mortality continues to grow exponentially with age even at very old ages. This observation may lead to more conservative estimates of future human longevity records.

\section{Acknowledgments}

The research reported in this publication was supported by the National Institute on Aging of the National Institutes of Health under Award No. R21AG054849 (to N.S.G.). The content is solely the responsibility of the authors and does not necessarily represent the official views of the National Institutes of Health.

\section{Statement of Ethics}

The authors have no ethical conflicts to disclose.

\section{Disclosure Statement}

The authors have no conflicts of interest to disclose.

\section{References}

1 Barbi E, Lagona F, Marsili M, Vaupel JW, Wachter KW. The plateau of human mortality: demography of longevity pioneers. Science. 2018 Jun;360(6396):1459-61.

2 Gavrilova NS, Gavrilov LA. Biodemography of old-age mortality in humans and rodents. J Gerontol A Biol Sci Med Sci. 2015 Jan; 70(1):1-9.

3 Gavrilov LA, Krut'ko VN, Gavrilova NS. The future of human longevity. Gerontology. 2017;63(6):524-6.

4 Vijg J, Le Bourg E. Aging and the inevitable limit to human life span. Gerontology. 2017; 63(5):432-4.

5 Greenwood M, Irwin JO. The biostatistics of senility. Hum Biol. 1939;11:1-23.

6 Horiuchi S, Wilmoth JR. Deceleration in the age pattern of mortality at older ages. Demography. 1998 Nov;35(4):391-412.

7 Thatcher AR. The long-term pattern of adult mortality and the highest attained age. J R Stat Soc Ser A Stat Soc. 1999;162(Pt. 1):5-43.

8 Thatcher AR, Kannisto V, Vaupel J. The force of mortality at ages 80 to 120 . Odense: Odense University Press; 1998.

9 Curtsinger JW, Fukui HH, Townsend DR, Vaupel JW. Demography of genotypes: failure of the limited life-span paradigm in Drosophila melanogaster. Science. 1992 Oct; 258(5081):461-3.

10 Gavrilov LA, Gavrilova NS. The biology of life span: a quantitative approach. New York: Harwood Academic Publisher; 1991.

11 Gavrilov LA, Gavrilova NS. Late-life mortality is underestimated because of data errors. PLoS Biol. 2019 Feb;17(2):e3000148.

12 Bronikowski AM, Alberts SC, Altmann J, Packer C, Carey KD, Tatar M. The aging baboon: comparative demography in a nonhuman primate. Proc Natl Acad Sci USA. 2002 Jul;99(14):9591-5.
13 Bronikowski AM, Altmann J, Brockman DK, Cords M, Fedigan LM, Pusey A, et al. Aging in the natural world: comparative data reveal similar mortality patterns across primates. Science. 2011 Mar;331(6022):1325-8.

14 Gavrilov LA, Gavrilova NS. Mortality Measurement at Advanced Ages: A Study of the Social Security Administration Death Master File. N Am Actuar J. 2011;15(3):432-47.

15 Bebbington M, Green R, Lai CD, Zitikis R Beyond the Gompertz law: exploring the late-life mortality deceleration phenomenon. Scand Actuar J. 2014;3:189-207.

16 Black DA, Hsu YC, Sanders SG, Schofield LS, Taylor LJ. The Methuselah effect: the pernicious impact of unreported deaths on oldage mortality estimates. Demography. 2017 Dec;54(6):2001-24.

17 Coale AJ, Li SM. The effect of age misreporting in China on the calculation of mortality rates at very high ages. Demography. 1991 May;28(2):293-301.

18 Gavrilova NS, Gavrilov LA. Mortality analysis of 1898-1902 birth cohort. Shaumburg (IL): Society of Actuaries; 2018.

19 Hill ME, Preston SH, Rosenwaike I. Age reporting among white Americans aged 85+: results of a record linkage study. Demography. 2000 May;37(2):175-86.

20 Yi Z, Vaupel JW. Oldest-old mortality in China. In: Robine JM, Crimmins E, Horiuchi S, Yi Z, editors. Individual life duration, and the growth of the oldest-old population. Dordrecht: Springer; 2007. p. 87-110.

21 Newman SJ. Errors as a primary cause of late-life mortality deceleration and plateaus. PLoS Biol. 2018 Dec;16(12):e2006776.

22 Preston SH, Elo IT. Effects of age misreporting on mortality estimates at older ages. Pop Stud. 1999;53(2):165-77.
23 Elo IT, Mykyta L, Sebastiani P, Christensen K, Glynn NW, Perls T. Age validation in the Long Life Family Study through a linkage to early-life census records. J Gerontol B Psychol Sci Soc Sci. 2013 Jul;68(4):580-5.

24 Dunn HL. Vital statistics of the United States 1957. Volume I. Washington; 1959.

25 University of California, Berkeley (USA) Max Planck Institute for Demographic Research (Germany). The Human Mortality Database [retrieved 2017 Apr 20]. Available from: www.mortality.org or www.humanmortality.de.

26 Kimball AW. Estimation of mortality intensities in animal experiments. Biometrics. 1960;16(4):505-21.

27 Chiang CL. Life table and mortality analysis. Geneva: World Health Organization; 1978.

28 Jdanov DA, Jasilionis D, Soroko EL, Rau R, Vaupel JW. Beyond the Kannisto-Thatcher database on old age mortality: an assessment of data quality at advanced ages. MPDIR working paper wp 2008-013. Rostock, Germany, MPDIR, 2008.

29 Young RD, Desjardins B, McLaughlin K, Poulain M, Perls TT. Typologies of extreme longevity myths. Curr Gerontol Geriatr Res. 2010;2010:423087.

30 Kannisto V. Development of oldest-old mortality, 1950-1990: evidence from 28 developed countries. Odense: Odense University Press; 1994.

31 Muller HG, Wang JL, Capra WB. From lifetables to hazard rates: the transformation approach. Biometrika. 1997;84(4):881-92.

32 StataCorp. Stata user's guide, release 14. College Station (TX): StataCorp LP; 2015.

33 Burnham KP, Anderson DR. Model selection and multimodel inference: a practical information-theoretic approach. New York: Springer-Verlag; 2002. 
34 Coale AJ, Kisker EE. Mortality crossovers reality or bad data. Pop Stud. 1986;40(3): 389-401.

35 Kestenbaum B, Ferguson BR. Supercentenarians in the United States. In: Maier $\mathrm{H}$, Gampe J, Jeune B, Robine JM, Vaupel JW, editors. Supercentenarians. Berlin: SpringerVerlag Berlin; 2010. p. 43-58.

36 Robine JM, Cubaynes S. Worldwide demography of centenarians. Mech Ageing Dev. 2017 Jul;165 Pt B:59-67.

37 Feehan DM. Separating the signal from the noise: evidence for deceleration in old-age death rates. Demography. 2018 Dec;55(6): 2025-44.
38 Wilmoth JR. Are mortality-rates falling at extremely high ages - an investigation based on a model proposed by Coale and Kisker. Pop Stud. 1995;49(2):281-95.

39 Beard RE. Note on some mathematical mortality models. In: Wolstenholme EW, O'Connor MO, editors. The lifespan of animals. Boston: Little, Brown; 1959. p. 302-11.

40 Vaupel JW, Manton KG, Stallard E. The impact of heterogeneity in individual frailty on the dynamics of mortality. Demography. 1979 Aug;16(3):439-54.
41 Wrigley-Field E. Mortality deceleration and mortality selection: three unexpected implications of a simple model. Demography. $2014 \mathrm{Feb} ; 51(1): 51-71$.

42 Lynch SM, Brown JS. Reconsidering mortality compression and deceleration: an alterative model of mortality rates. Demography. 2001 Feb;38(1):79-95.

43 Engelman M, Canudas-Romo V, Agree EM. The implications of increased survivorship for mortality variation in aging populations. Popul Dev Rev. 2010;36(3):511-39. 